\title{
Desenvolvimento de snacks extrusados a partir de misturas de farinha de soja, fécula e farelo de mandioca
}

\author{
Development of extruded snacks from blends of soya flour, cassava starch and bran
}

\author{
Fernanda Rossi Moretti Trombini ${ }^{\mathrm{I}}$ Magali Leonel ${ }^{\mathrm{II}}$ Martha Maria Mischan $^{\mathrm{III}}$
}

\section{RESUMO}

Neste trabalho, objetivou-se avaliar o efeito das condições de extrusão sobre as propriedades físicas de produtos extrusados. Foi utilizado o delineamento central composto rotacional com quatro variáveis independentes $\left(2^{4}\right)$ e a metodologia de superfície de resposta para avaliar os resultados de índice de expansão, volume específico, índice de absorção de água, indice de solubilidade em água e cor, de acordo com as variações de temperatura de extrusão, rotação da rosca, porcentagem de farelo de mandioca e porcentagem de farinha de soja. Os resultados indicam que é possível produzir novos produtos extrusados com boas propriedades físicas a partir de misturas de farinha de soja, fécula e farelo de mandioca.

Palavras-chave: extrusão, amido, expansão, cor, água.

\begin{abstract}
This study aimed to evaluate the effect of the extrusion conditions on physical properties of extruded products. A factorial central composite design $\left(2^{4}\right)$ with four independent variables and the response surface methodology were used to evaluate the results of expansion index, specific volume, water absorption index, water solubility index and color, according to the variations in barrel temperature, screw speed, percentage of cassava bran and percentage of soya flour. The results indicate that is possible to produce new extruded products with good physical properties from blends of soya flour, cassava starch and bran.
\end{abstract}

Key words: extrusion, starch, expansion, color, water.

\section{INTRODUÇÃO}

A mandioca é uma tuberosa amilácea de grande potencial energético, podendo ser utilizada tanto para o consumo in natura como para industrialização. As unidades de processamento da mandioca para produção de fécula e polvilho azedo produzem um material sólido identificado por farelo, bagaço ou polpa residual, o qual tem sido subaproveitado na alimentação de animais. Esse resíduo sólido é composto pelo material fibroso da raiz, contendo parte do amido que não foi possível extrair no processamento. É gerado na etapa de separação da fécula e, por embeber-se de água, em volume, apresenta maior quantidade que a própria matéria-prima, contendo 75\% de umidade (LEONEL, 2001).

Estudos revelam os benefícios dos alimentos funcionais, o que tem despertado grande interesse de consumidores que desejam incorporar na sua dieta produtos ricos em componentes nutricionais que, além de nutrir, ainda são capazes de proteger o organismo contra doenças. Nos últimos anos, a procura por alimentos derivados de soja tem aumentado, devido à divulgação dos benefícios à saúde atribuídos ao consumo dessa leguminosa. O processamento da soja dá origem a diferentes matériasprimas como farinhas de soja, extratos hidrossolúveis e proteínas texturizadas, que podem ser utilizados na

\footnotetext{
'Programa de Pós-graduação em Energia na Agricultura, Faculdade de Ciências Agronômicas (FCA), Universidade Estadual Paulista "Júlio de Mesquita Filho" (UNESP), Botucatu, SP, Brasil.

"Centro de Raízes e Amidos Tropicais (CERAT), UNESP, Rua José Barbosa de Barros, 1780, 18610-307, Botucatu, SP, Brasil. E-mail: mleonel@cerat.unesp.br. Autor para correspondência.

"IIDepartamento de Bioestatística, Instituto de Biociências (IB), UNESP, Botucatu, SP, Brasil.
} 
produção de alimentos que fazem parte da dieta ocidental (GENOVESE \& LAJOLO, 2002).

O mercado de snacks no Brasil, especificamente o mercado de "salgadinhos", apresenta um faturamento anual de aproximadamente $\mathrm{R} \$ 1$ bilhão, com produção em torno de 80 mil toneladas, apresentando crescimento das linhas de produtos com apelos nutricionais, como os fortificados, com baixo teor de gordura, e os funcionais (FERREIRA, 2006).

A tecnologia de extrusão vem sendo explorada nos últimos anos para o desenvolvimento de novos produtos alimentícios. Essa tecnologia possibilita, com pouca ou nenhuma modificação dos equipamentos básicos, um controle apropriado do processo e a produção de uma grande variedade de produtos (CHANG, 2001).

Para o processo de extrusão, mudanças nos ingredientes ou parâmetros, como velocidade e temperatura, podem afetar as variáveis do sistema de extrusão e as características do produto, como: textura, estrutura, grau de expansão e atributos sensoriais (MENDONÇA et al., 2000).

Diante do grande interesse do setor agroindustrial da mandioca na implantação de novas tecnologias para o desenvolvimento de produtos e aproveitamento dos resíduos, neste trabalho, objetivou-se avaliar o efeito da composição da matériaprima, da temperatura de extrusão e da rotação da rosca sobre as propriedades físicas de produtos extrusados obtidos a partir de misturas de farelo e fécula de mandioca com farinha de soja.

\section{MATERIAL E MÉTODOS}

Foram utilizadas como matérias-primas a farinha de soja integral (Jasmine), a fécula e o farelo de mandioca (Lotus Comércio e Indústria de Produtos de Mandioca Ltda). As matérias-primas foram caracterizadas quanto à umidade, proteína, lipídeos, cinzas, fibras, açúcares totais e amido (AACC, 1983).

A extrusão foi efetuada em uma linha completa de extrusão INBRA RX50 da Inbramaq S/A, com sistema de extrusão através de fricção mecânica, rosca simples de extrusão, sistema de controle de temperatura e velocidade variável.

O processo de extrusão teve como parâmetros fixos: taxa de compressão da rosca (3:1); comprimento da rosca $(45 \mathrm{~cm})$, diâmetro da rosca $(1,9 \mathrm{~cm})$; taxa de alimentação $\left(180 \mathrm{~g} \mathrm{~min}^{-1}\right)$; abertura da matriz $(4 \mathrm{~mm})$; temperatura na $1^{1}$ zona $\left(25^{\circ} \mathrm{C}\right)$ e na $2^{2}$ zona $\left(40^{\circ} \mathrm{C}\right)$; e porcentagem de umidade nas misturas $(15 \%)$. Foram consideradas como variáveis independentes: a temperatura de extrusão (3aㅡ zona), a rotação da rosca, a porcentagem de farelo de mandioca e a de farinha de soja em mistura com a fécula de mandioca.

Para analisar o efeito combinado das variáveis independentes nas características dos extrusados, foi utilizado o delineamento central composto rotacional para quatro fatores, totalizando 25 tratamentos: dezesseis tratamentos correspondentes ao fatorial $2^{4}$, em que os quatro fatores são: $\mathrm{T}=$ temperatura na $3^{\mathrm{a}}$ zona $\left({ }^{\circ} \mathrm{C}\right), \mathrm{R}=$ rotação da rosca $(\mathrm{rpm})$, farinha de soja (FS) e farelo de mandioca (FM), cada qual em dois níveis, codificados como -1 e +1 ; oito tratamentos com os níveis mínimo e máximo de cada fator, codificados como - $\alpha e+\alpha$, respectivamente, um tratamento central repetido seis vezes, em que os fatores estão todos em um nível médio, codificado como zero, a fim de estimar o erro experimental (Tabela 1).

Foram preparados $700 \mathrm{~g}$ de misturas com granulometria de $0,25 \mathrm{~mm}$ em homogenizador em " $\mathrm{Y}$ " (TE 201/5 Tecnal) nas proporções do delineamento experimental.

As misturas foram analisadas quanto aoíndice de solubilidade em água e índice de absorção de água (ANDERSON et al., 1969), bem como, para os parâmetros de cor. A cor foi avaliada em colorímetro Minolta CR-400, com a utilização de iluminante D65, sendo feitas leituras de três amostras. Os resultados foram expressos, no sistema CIELAB, em valores L*, a* e b*.

O índice de expansão dos extrusados foi calculado pela relação entre o diâmetro da amostra e o diâmetro da matriz. $\mathrm{O}$ valor considerado foi obtido pela média aritmética das medidas de 20 diferentes produtos expandidos dentro de cada tratamento (FAUBION \& HOSENEY, 1982).

O volume específico dos produtos expandidos foi determinado pelo método do deslocamento da massa ocupada (semente de painço) e determinado o seu volume em uma proveta graduada. Os resultados foram obtidos pela média aritmética das medidas de 20 diferentes produtos por tratamento e expressos em $\mathrm{mLg}^{-1}$ (FAUBION \& HOSENEY, 1982).

Tabela 1 - Parâmetros variáveis do processo de extrusão.

\begin{tabular}{|c|c|c|c|c|c|}
\hline Axiais & Codificados & FM & FS & $\mathrm{T}$ & RR \\
\hline \multirow[t]{4}{*}{$-\alpha$} & -2 & 10 & 10 & 45 & 190 \\
\hline & -1 & 15 & 15 & 60 & 210 \\
\hline & 0 & 20 & 20 & 75 & 230 \\
\hline & +1 & 25 & 25 & 90 & 250 \\
\hline$+\alpha$ & +2 & 30 & 30 & 105 & 270 \\
\hline
\end{tabular}

FM: farelo de mandioca (\%); FS: Farinha de soja (\%); T: Temperatura de extrusão $\left({ }^{\circ} \mathrm{C}\right) ; \mathrm{RR}$ : rotação da rosca $(\mathrm{rpm})$. 


\section{RESULTADOS E DISCUSSÃO}

Análises das matérias-primas e das misturas

A composição química das matérias-primas está entre os principais fatores interferentes no desenvolvimento de produtos extrusados. A fécula de mandioca apresentou baixas quantidades de componentes não amido com composição próxima à observada por LEONEL et al. (2009) (Tabela 2). A farinha de soja apresentou elevado teor proteico e, também, considerável teor de matéria-graxa e fibras (Tabela 2). Esses resultados encontraram-se próximos aos relatados por SILVA et al. (2006) para grãos de soja que foram de 5,6\% de umidade, 40,4\% de proteína, $24,55 \%$ de lipídios, $17,26 \%$ de carboidratos, $9,31 \%$ de fibras e $2,88 \%$ de cinzas. $\mathrm{O}$ farelo de mandioca apresentou teor de fibra superior ao relatado por LEONEL et al. (2008), que, analisando farelo obtido em indústria de polvilho azedo, observaram 9,9\% de fibras. Essa diferença pode ser devido ao processo mais eficiente de extração de amido das grandes fecularias, gerando resíduo com maior teor de fibras (Tabela 2).

A análise de cor das misturas antes da extrusão mostrou variação de 88,2 a 91,0 para a luminosidade, de 0,14 a 0,89 para o croma a* e de 9,39 a 12,03 para o croma $b^{*}$.

Os índices de solubilidade (ISA) variaram de 2,78 a $6,81 \%$ nas misturas antes do processo de extrusão. O maior valor de ISA foi obtido com $30 \%$ de farinha de soja na mistura, o que se deve provavelmente ao grande teor de proteína presente nessa mistura.

Os índices de absorção de água das misturas antes do processo de extrusão variaram de $2,92 \mathrm{~g} \mathrm{gel} \mathrm{g}^{-1}$ a 2,35g gel g-1, com o menor valor observado na mistura com maior porcentagem de fécula.

Análises dos produtos extrusados

No desenvolvimento de produtos extrusados expandidos, são parâmetros de qualidade desejados: elevados índices de expansão, baixa densidade, elevado volume específico, cor clara e elevada crocância (LEONEL et al., 2010).

A análise de cor dos produtos extrusados mostrou que, após o processo, a luminosidade dos produtos variou de 64,11 a 75,25 , mostrando ter ocorrido escurecimento, independente do tratamento utilizado. A análise dos dados evidenciou efeito da porcentagem de farelo de mandioca, temperatura e rotação, e das interações entre o farelo de mandioca com farinha de soja e temperatura com rotação (Tabela 3 ).

Nas condições de baixa temperatura de extrusão e elevada rotação testadas, observaram-se menores valores de luminosidade, ou seja, produtos extrusados mais escuros. Além disso, a luminosidade é menor quando o teor de farinha de soja e farelo de mandioca estão presentes na mistura em maior proporção (Figura 1).

ILO \& BERGHOFER (1999) avaliaram diferentes parâmetros do processo de extrusão em produtos a base de milho e concluíram que os únicos parâmetros significativos na análise de cor foram a temperatura de processo e a umidade inicial das matérias-primas. Valores de $\mathrm{L}^{*}$ dos extrusados diminuíram com o aumento da temperatura $\left(140 \mathrm{a} 180^{\circ} \mathrm{C}\right)$ e aumentaram ao se elevar a umidade (13 a 17\%). Os valores observados pelos autores foram de 73,00 a 86,17, valores superiores aos obtidos neste trabalho.

Para o croma $a^{*}$, foi observada uma variação de 5,45 a 3,57, valores estes superiores aos observados nas misturas antes da extrusão. A análise dos dados mostrou terem ocorrido efeitos da porcentagem de farelo de mandioca e temperatura, bem como das interações de farelo de mandioca com rotação e temperatura e rotação (Tabela 3). Nas condições de baixa temperatura e rotação e também nos seus inversos, o croma a* é menor nos produtos extrusados. Nas condições de maior teor de farelo de mandioca na mistura e baixa rotação, são encontrados maiores valores do croma $\mathrm{a}^{*}$ (Figura 1$)$.

Tabela 2 - Composição média da fécula, farelo de mandioca e farinha de soja.

\begin{tabular}{|c|c|c|c|}
\hline Análises & Fécula & Farelo & Farinha de soja \\
\hline Umidade $\left({\left.\mathrm{g} 100 \mathrm{~g}^{-1}\right)}^{-1}\right.$ & $13,8 \pm 0,04$ & $6,4 \pm 0,03$ & $7,2 \pm 0,06$ \\
\hline 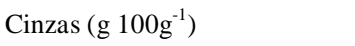 & $0,07 \pm 0,006$ & $1,57 \pm 0,01$ & $0,79 \pm 0,02$ \\
\hline Fibras $\left({\left.\mathrm{g} 100 \mathrm{~g}^{-1}\right)}^{1}\right.$ & $0,39 \pm 0,06$ & $21,37 \pm 1,22$ & $8,83 \pm 0,21$ \\
\hline 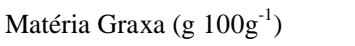 & $0,11 \pm 0,02$ & $0,34 \pm 0,02$ & $21,33 \pm 0,17$ \\
\hline Proteínas $\left({\left.\mathrm{g} 100 \mathrm{~g}^{-1}\right)}^{-1}\right.$ & $0,44 \pm 0,04$ & $2,58 \pm 0,28$ & $46,75 \pm 0,75$ \\
\hline Carboidratos Totais $\left({\left.\mathrm{g} 100 \mathrm{~g}^{-1}\right)}^{-1}\right.$ & $85,19 \pm 0,17$ & $67,74 \pm 1,56$ & $15,1 \pm 1,21$ \\
\hline Acidez Titulável & $1,36 \pm 0,02$ & $12,17 \pm 1,03$ & $3,20 \pm 0,12$ \\
\hline $\mathrm{pH}$ & $5,38 \pm 0,06$ & $5,19 \pm 0,05$ & $6,95 \pm 0,04$ \\
\hline
\end{tabular}

Ciência Rural, v.43, n.1, jan, 2013. 
Tabela 3 - Coeficientes de regressão estimados (modelo $\mathrm{y}_{\mathrm{k}}=\beta_{0}+\beta_{1} \mathrm{x}_{1}+\beta_{2} \mathrm{x}_{2}+\beta_{3} \mathrm{x}_{3}+\beta_{4} \mathrm{x}_{4}+\beta_{12} \mathrm{x}_{1} \mathrm{x}_{2}+\beta_{13} \mathrm{x}_{1} \mathrm{x}_{3}+\beta_{14} \mathrm{x}_{1} \mathrm{x}_{4}+\beta_{23} \mathrm{x}_{2} \mathrm{x}_{3}+\beta_{24} \mathrm{x}_{2} \mathrm{x}_{4}+\beta_{34} \mathrm{x}_{3} \mathrm{x}_{4}$ )

\begin{tabular}{|c|c|c|c|c|c|c|c|}
\hline \multirow{2}{*}{ Parâmetros } & \multicolumn{7}{|c|}{--Variáveis dependentes- } \\
\hline & IE & VE & ISA & IAA & $\mathrm{L}^{*}$ & $a^{*}$ & $b^{*}$ \\
\hline$\beta_{0}$ & 2,08 & 4,39 & 12,5 & 7,16 & 71,9 & 4,45 & 21,9 \\
\hline$\beta_{1}(\mathrm{FM})$ & $\mathrm{ns}$ & $\mathrm{ns}$ & $\mathrm{ns}$ & ns & $-1,3 * * *$ & $0,4 * * *$ & $0,52^{*}$ \\
\hline$\beta_{2}(\mathrm{FS})$ & $\mathrm{ns}$ & $\mathrm{ns}$ & $\mathrm{ns}$ & $-0,46^{*}$ & $\mathrm{~ns}$ & $\mathrm{~ns}$ & $\mathrm{~ns}$ \\
\hline$\beta_{3}(\mathrm{~T})$ & $\mathrm{ns}$ & $\mathrm{ns}$ & $2,62 * * *$ & $0,48 * *$ & $1,56^{* * *}$ & $-0,19 *$ & $\mathrm{~ns}$ \\
\hline$\beta_{4}(\mathrm{RR})$ & $\mathrm{ns}$ & $\mathrm{ns}$ & $\mathrm{ns}$ & ns & $-0,8^{* * *}$ & $\mathrm{~ns}$ & $\mathrm{~ns}$ \\
\hline$\beta_{11}\left(\mathrm{FM}^{2}\right)$ & $0,05^{*}$ & $\mathrm{~ns}$ & $1,95^{* *}$ & $\mathrm{~ns}$ & ns & $\mathrm{ns}$ & ns \\
\hline$\beta_{22}\left(\mathrm{FS}^{2}\right)$ & $0,07 * *$ & $\mathrm{~ns}$ & $\mathrm{~ns}$ & $\mathrm{~ns}$ & $\mathrm{~ns}$ & $\mathrm{~ns}$ & $\mathrm{~ns}$ \\
\hline$\beta_{33}\left(T^{2}\right)$ & $0,06^{*}$ & $0,98 * * *$ & $1,46^{*}$ & $\mathrm{~ns}$ & $\mathrm{~ns}$ & $\mathrm{~ns}$ & $0,44^{*}$ \\
\hline$\beta_{44}\left(\mathrm{RR}^{2}\right)$ & $0,05^{* *}$ & ns & $\mathrm{ns}$ & $\mathrm{ns}$ & $\mathrm{ns}$ & $\mathrm{ns}$ & $\mathrm{ns}$ \\
\hline$\beta_{12}(\mathrm{FM} * \mathrm{FS})$ & $\mathrm{ns}$ & $\mathrm{ns}$ & $\mathrm{ns}$ & $\mathrm{ns}$ & $-0,68^{*}$ & $\mathrm{~ns}$ & $-0,61 *$ \\
\hline$\beta_{13}\left(\mathrm{FM}^{* \mathrm{~T}}\right)$ & $\mathrm{ns}$ & $\mathrm{ns}$ & $\mathrm{ns}$ & $\mathrm{ns}$ & $\mathrm{ns}$ & $\mathrm{ns}$ & $\mathrm{ns}$ \\
\hline$\beta_{14}(\mathrm{FM} * \mathrm{RR})$ & $\mathrm{ns}$ & $\mathrm{ns}$ & $\mathrm{ns}$ & $\mathrm{ns}$ & $\mathrm{ns}$ & $-0,21 *$ & $\mathrm{~ns}$ \\
\hline$\beta_{23}(\mathrm{FS} * \mathrm{~T})$ & $\mathrm{ns}$ & $\mathrm{ns}$ & $\mathrm{ns}$ & $\mathrm{ns}$ & $\mathrm{ns}$ & $\mathrm{ns}$ & ns \\
\hline$\beta_{24}(\mathrm{FS} * \mathrm{RR})$ & $-0,1 * * *$ & $-0,49 *$ & $\mathrm{~ns}$ & $\mathrm{~ns}$ & $\mathrm{~ns}$ & $\mathrm{~ns}$ & $\mathrm{~ns}$ \\
\hline$\beta_{34}\left(\mathrm{~T}^{*} \mathrm{RR}\right)$ & $0,06^{*}$ & $\mathrm{~ns}$ & $\mathrm{~ns}$ & $0,41 *$ & $1,1 * * *$ & $-0,26 * *$ & $\mathrm{~ns}$ \\
\hline $\mathrm{R}^{2}$ & 0,64 & 0,55 & 0,51 & 0,43 & 0,83 & 0,66 & 0,31 \\
\hline F valor & 6,78 & 16,46 & 9,01 & 6,49 & 24,06 & 12,41 & 3,96 \\
\hline $\operatorname{Pr}>F$ & 0,0003 & $<0,0001$ & 0,0003 & 0,002 & $<0,0001$ & $<0,0001$ & 0,019 \\
\hline
\end{tabular}

$\beta_{1}(\mathrm{FM})=$ farelo de mandioca (linear) ; $\beta_{2}(\mathrm{FS})=$ farinha de soja (linear); $\beta_{3}(\mathrm{~T})=$ temperatura (linear); $\beta_{4}(\mathrm{RR})=$ rotação da rosca (linear); $\beta_{11}\left(\mathrm{FM}^{2}\right)=$ farelo de mandioca (quadrático); $\beta_{22}\left(\mathrm{FS}^{2}\right)=$ farinha de soja (quadrático); $\beta_{33}\left(\mathrm{~T}^{2}\right)=$ temperatura (quadrático); $\beta_{44}\left(\mathrm{RR}^{2}\right)=$ rotação da rosca (quadrático); $\mathrm{R}^{2}=$ coeficiente de determinação; ns= não significativo; $*=\mathrm{P}<0,05, * *=\mathrm{P}<0,01, * * *=\mathrm{P}<0.001$; IE=índice de expansão; $\mathrm{VE}=$ volume específico $\left(\mathrm{mL} \mathrm{g}^{-1}\right)$; ISA=índice de solubilidade em água $(\%) ; \mathrm{IAA}=$ índice de absorção de água; $\mathrm{L}^{*}=$ luminosidade; $\mathrm{a}^{*}=$ croma $\mathrm{a} ; \mathrm{b}^{*}=$ croma $\mathrm{b}$.

A variação observada para o croma $b *$ nos diferentes tratamentos foi de 20,43 a 26,45 , o que evidencia o aumento da presença deste croma com o processo de extrusão. A análise estatística mostrou a influência da porcentagem de farelo de mandioca, da temperatura de extrusão e da interação de farelo de mandioca e farinha de soja sobre este parâmetro de cor (Tabela 3). Nas condições intermediárias de temperatura o croma b* é menor, e maior na condição de elevado teor de farelo de mandioca e baixa concentração de farinha de soja (Figura 1).

As condições de processamento utilizadas na extrusão (alta temperatura e baixa umidade) são reconhecidas por favorecer a reação entre açúcares redutores e aminoácidos, o que resulta na formação de compostos coloridos e na redução do aminoácido lisina. $\mathrm{Se}$ o escurecimento é muito intenso, cores e sabores indesejáveis podem aparecer (ILO \& BERGHOFER, 1999).

Os resultados obtidos para o índice de absorção de água dos extrusados (IAA) variaram de 5,8 a $8,82 \mathrm{~g} \mathrm{gel} \mathrm{g}^{-1}$. A análise dos dados mostrou ter havido influência dos fatores: farinha de soja, temperatura e interação temperatura e rotação (Tabela 3). Nas condições de elevado teor de farinha de soja e baixa temperatura, são obtidos menores IAA (Figura 1).

Os resultados obtidos para o índice de solubilidade em água (ISA), nos diferentes tratamentos após a extrusão, mostraram uma variação de 8,93 a $25,2 \%$. Os coeficientes de regressão evidenciaram efeitos significativos para a temperatura e farelo de mandioca (Tabela 3).

Nas condições centrais do delineamento experimental dos fatores temperatura $\left(75^{\circ} \mathrm{C}\right)$ e porcentagem de farelo de mandioca (20\%) são obtidos os menores valores para ISA. Já nas condições extremas opostas de farelo de mandioca e temperatura, são obtidos os maiores índices de absorção de água (Figura 1).

MESA et al. (2009), estudando o efeito da rotação da rosca e porcentagem de concentrado proteico de soja na produção de snacks fortificados de amido de milho, observaram o efeito dos dois fatores sobre os IAA e ISA dos produtos, com baixo valor de IAA nas condições de alta rotação da rosca e maiores porcentagens de concentrado proteico de soja. Já o ISA foi maior nas condições de elevada rotação (330rpm) e alto conteúdo de concentrado de soja (20\%). 


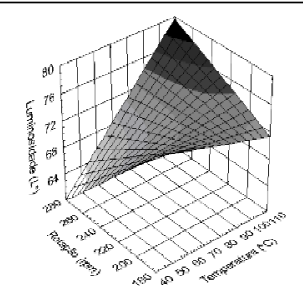

(a)

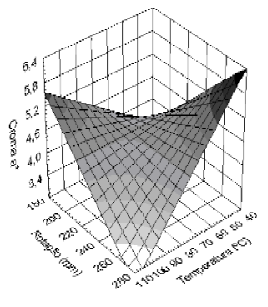

(c)

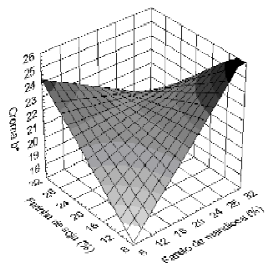

(e)
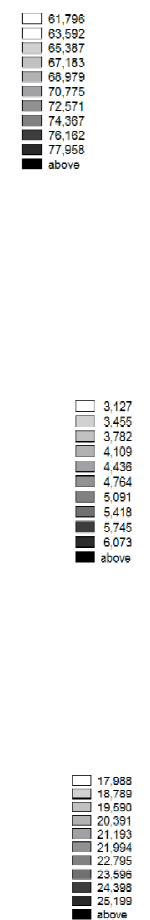

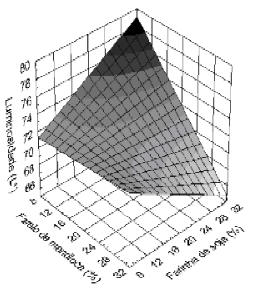

(b)

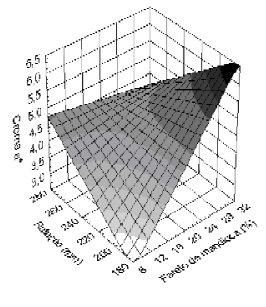

(d)

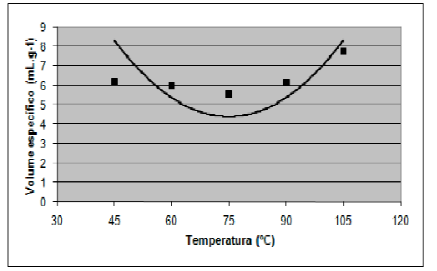

(f)

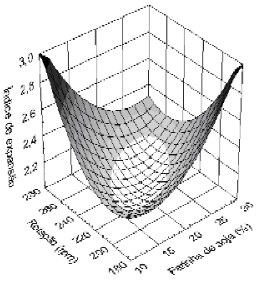

(g)

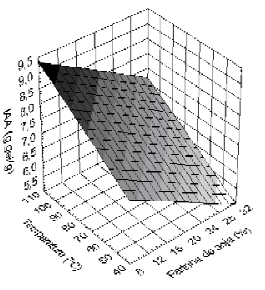

(i)
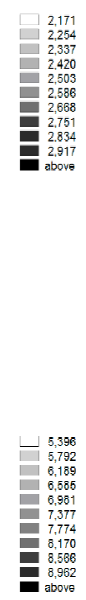

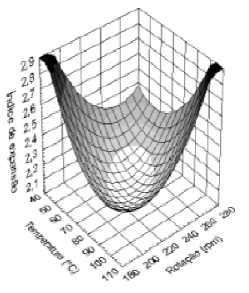

(h)

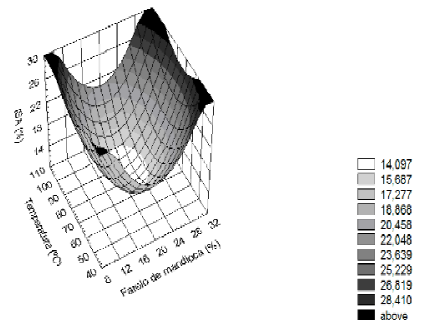

(j)

Figura 1 - Efeitos da temperatura, rotação da rosca, porcentagem de farelo de mandioca e porcentagem de farinha de soja sobre as características dos produtos extrusados: (a, b) luminosidade; (c,d) croma $\mathrm{a}^{*}$; (e) croma $\mathrm{b}^{*}$, .(f) volume específico; $(\mathrm{g}, \mathrm{h})$ índice de expansão; (i) índice de absorção de água (IAA); (j) índice de solubilidade em água (ISA). 
O volume específico é uma medida da expansão volumétrica, que é a soma das expansões radial e axial. Os valores observados para o volume específico dos produtos extrusados nas diferentes condições experimentais variaram de 2,64 a $7,75 \mathrm{ml} \mathrm{g}^{-1}$. A análise dos dados mostrou efeito quadrático da temperatura de extrusão, bem como da interação da porcentagem de farinha de soja na mistura e rotação da rosca, sobre este parâmetro (Tabela 3 ). Nas condições intermediárias de temperatura, o volume específico é menor (Figura 1).

SOUZA et al. (2007), avaliando o efeito das condições de extrusão em produtos expandidos, produzidos a partir de misturas de fécula de mandioca e polpa cítrica, observaram que o volume específico dos extrusados decresceu com o aumento da temperatura e umidade, variando de a 1,71 a $8,54 \mathrm{ml} \mathrm{g}^{-1}$.

Uma propriedade dos produtos extrusados é a expansão. A alta pressão existente próxima à descarga da matriz é reduzida quando o produto sai do extrusor, ocasionando a evaporação instantânea da água e a expansão do produto (BORBA et al., 2005). Os resultados obtidos para o índice de expansão dos extrusados variaram de 2,12 a 2,56 nos diferentes tratamentos. A análise dos coeficientes de regressão mostrou terem ocorrido efeitos de todas as variáveis independentes (Tabela 3). Observou-se que, nas condições extremas, opostas de farinha de soja e rotação, bem como, nas condições de baixa rotação e temperatura e nos seus inversos, foram obtidos os maiores índices de expansão (Figura 1).

HASHIMOTO \& GROSSMANN (2003), analisando o efeito das condições de extrusão sobre a qualidade de extrusados de misturas de farelo e amido de mandioca, obtiveram índice de expansão variando de 1,6 a 3,2, e observaram a influência da porcentagem de fibras na expansão dos produtos.

\section{CONCLUSÃO}

Os resultados obtidos neste estudo mostram que o processo de extrusão afeta as propriedades físicas dos extrusados. A composição química das matérias-primas está em acordo com o esperado. As misturas extrusadas apresentam aumento da solubilidade e absorção de água. Parâmetros desejados em snacks, como maior expansão e menor escurecimento, são obtidos nas condições de elevada temperatura de extrusão $\left(90-105^{\circ} \mathrm{C}\right)$ e rotação da rosca (270rpm), com as porcentagens intermediárias de farelo de mandioca (20\%) e baixa porcentagem de farinha de soja $(10 \%)$, misturados à fécula de mandioca.

\section{REFERÊNCIAS}

AMERICAN ASSOCIATION OF CEREAL CHEMISTS. Approved methods of the American Association of Cereal Chemists. 8.ed. St. Paul, 1983. 1094p.

ANDERSON R.A. et al. Gelatinization of corn grits by roll-and extrusion-cooking. Cereal Science Today, Minneapolis, v.14, n.1, p.4-7, 1969.

BORBA, A.M. et al. Efeito dos parâmetros de extrusão sobre as propriedades funcionais de extrusados da farinha de batata doce. Ciência e Tecnologia de Alimentos, Campinas, v.25, n.4, p.835-843, 2005. Disponível em: <http://www.scielo.br/pdf/ cta/v25n4/27660.pdf>. Acesso em: 02 maio, 2011.

CHANG, Y.K. Influence of extrusion conditions on cassava starch and soybean protein concentrate blends. Acta Alimentaria, Budapest, v.30, n.2, p.189-203, 2001. Disponível em: <http://www.akademiai.com/content/119693/>. Acesso em 26 jan. 2011.

FAUBION, J.M.; HOSENEY, R.C. High temperature and short time. Extrusion-cooking of wheat starch and flour. I- Effect of moisture and flour type on extrudate properties. Cereal Chemistry, St Paul, v.59, n.6, p.529-533, 1982. Disponível em: <http://www.aaccnet.org/cerealchemistry/Contents/ NovDec82.asp>. Acesso em: 25 jan. 2011.

FERREIRA, R.E. Avaliação de parâmetros do processo de extrusão e do teor de farelo de trigo adicionado em características de "snacks" expandidos de milho. 2006. 162f. Tese (Doutorado em Tecnologia de Alimentos) Faculdade de Engenharia de Alimentos, Universidade Estadual de Campinas, SP.

GENOVESE, M.I.; LAJOLO, F.M. Isoflavones in soy based foods consumed in Brazil: levels, distribution, and estimated intake. Journal of Agricultural and Food Chemistry, Washington, v.50, p.5987-5993, 2002. Disponível em: <http:/ /pubs.acs.org/doi/pdfplus/10.1021/jf0202990>. Acesso em: 02 maio, 2011.

HASHIMOTO, J.M.; GROSSMANN, M.V.E. Effects of extrusion conditions on quality of cassava bran/cassava starch extrudates. International Journal of Food Science and Technology, Oxford, v.38, n.5, p.511-517, 2003. Disponível em: <http://www3.interscience.wiley.com/cgi-bin/fulltext/ 118888399/PDFSTART>. Acesso em: 25 abr. 2011.

ILO, S.; BERGHOFER, E. Kinetics of colour changes during extrusion cooking of maize grits. Journal of Food Engineering, Oxford, v.39, n.1, p.73-80, 1999. Disponível em: <http:// www.sciencedirect.com/science/article/pii/ S0260877498001484>. Acesso em: 19 abr. 2011.

LEONEL, M. Caracterização da fibra e uso do farelo de mandioca como base para produtos dietéticos. In: ____. Manejo, uso e tratamento de subprodutos da industrialização da mandioca. São Paulo: Fundação Cargill, Série Culturas de Tuberosas Amiláceas Latino Americanas, 2001. p.221-226.

LEONEL, M. et al. Produção de biscoitos extrusados de polvilho azedo com fibras: efeito de parâmetros operacionais sobre as propriedades físicas. Ciência e Tecnologia de Alimentos, Campinas, v.28, n.3, p.586-591, 2008. Disponível em: <http:/ 
/www.scielo.br/pdf/cta/v28n3/a13v28n3.pdf>. Acesso em: 16 abr. 2011.

LEONEL, M. et al. Physical characteristics of extruded cassava starch. Scientia Agricola, Piracicaba, v.66, n.4, p.486-493, 2009. Disponível em: <http://www.scielo.br/pdf/sa/v66n4/ a09v66n4.pdf>. Acesso em: 19 abr. 2011.

MENDONÇA, S. et al. Corn bran as a fibre source in expanded snacks. Lebensmittel Wissenschaft and Technologie, Zürich, v.33, n.1, p.2-8, 2000. Disponível em: <http:// www.sciencedirect.com/science/article/pi i/ S0023643899906011>. Acesso em: 24 jan. 2011.

MESA, N.J.E. et al. Soy protein-fortified expanded extrudates. Baseline study using normal corn starch. Journal of Food

Engineering, Oxford, v.90, p.262-270, 2009. Disponível em: <http://www.sciencedirect.com/science/article/pii/ S0260877408003294>. Acesso em:11 abr. 2011.

SILVA, M.S. et al. Composição química e valor protéico do resíduo de soja em relação ao grão de soja. Ciência e Tecnologia de Alimentos, Campinas, v.26, n.3, p.571-576, 2006. Disponível em: <http://www.scielo.br/pdf/cta/v26n3/ 31758.pdf $>$. Acesso em: 15 abr. 2011.

SOUZA, L.B. et al. Efeito dos parâmetros de extrusão nas propriedades físicas de extrusados de misturas de fécula de mandioca e polpa cítrica. Alimentos e Nutrição. Araraquara, v.18, p.83-91, 2007. Disponível em: <http://200.145.71.150/ seer/index.php/alimentos/article/view/138/147>. Acesso em: 15 abr. 2011 